\section{Prior supplementation with long chain omega-3 polyunsaturated fatty acids promotes weight loss in obese adults: a double-blinded randomised controlled trial}

\begin{abstract}
Irene A. Munro and Manohar L. Garg*
Obesity has been linked with low levels of $\omega-3$ fatty acids. Generally, intervention studies have failed to establish benefits of supplementation with $\omega$-3PUFA in reducing body weight or fat mass in humans. The aim of this study was to investigate whether supplementation with LC $\omega$-3PUFA alone, then consumed concomitantly with a very low energy diet (VLED), facilitated weight loss, improvements in blood lipids and positive changes to inflammatory mediators. This was a double blind randomised controlled trial with two parallel groups. For 4 weeks of prior supplementation, one group consumed 6 $\times 1 \mathrm{~g}$ capsules per day monounsaturated oil (placebo), the other group consumed $6 \times 1 \mathrm{~g}$ capsules per day LC $\omega$-3PUFA (fish oil) each comprising $70 \mathrm{mg}$ EPA and $270 \mathrm{mg}$ DHA, while consuming their usual diet. Each group continued with their supplements for another 4 weeks while both groups followed a VLED regimen ( $n=19$ placebo, $n=20$ fish oil). Fasting blood samples, anthropometric measurements and 3-day food diaries were collected at baseline, at 4 weeks and at 8 weeks. At 4 weeks levels of EPA and DHA increased two-fold in the fish oil group $(P<0.001)$, with no significant changes to anthropometric measurements for either group. At 8 weeks a significant 3-way interaction between time, group and gender was observed for percentage reduction in weight, $F(1,35)=5.55, P=0.024$, and $\mathrm{BMI}, F(1,35)=5.3, P=0.027$ with a greater percentage decrease for females in $\mathrm{FO}$ compared to $\mathrm{PB}$ for weight $(-7.21 \%$ vs. $-5.82 \%)$ and $\mathrm{BMI}(-7.43 \%$ vs. $-5.91 \%)$ respectively $(P<0.05$ for both). It would appear that supplementation with LC $\omega$-3PUFA had a time dependent effect on weight loss in females.
\end{abstract}

Received 25th January 2013 Accepted 1st February 2013

DOI: $10.1039 / \mathrm{c} 3 \mathrm{fo} 60038 \mathrm{f}$

www.rsc.org/foodfunction effects. $^{\mathbf{1 3 , 1 4}}$ Similarly, there is conflicting evidence on the effects of supplementation with LC $\omega$-3PUFA on adiposity in humans. In some studies LC $\omega$-3PUFA has been associated with weight loss, ${ }^{15,16}$ in others with a decrease in adipose tissue mass, ${ }^{\mathbf{1 7}, 18}$ while other studies have reported no effect on adiposity. ${ }^{5,19}$

It has also been reported that concentrations of LC $\omega$-3PUFA are significantly lower in obese compared to normal weight adults, ${ }^{20}$ adolescents, ${ }^{21,22}$ and children ${ }^{23}$ suggesting that high concentrations of LC $\omega$-3PUFA in the body inhibit the development of adiposity or have possibly reduced weight previously gained. Is it, therefore, possible that if obese individuals are given time to increase levels of LC $\omega$-3PUFA prior to commencing a reduced energy weight loss diet, it might result in greater weight reduction. Thus the main aim of this study was to examine whether prior supplementation with LC $\omega$ 3PUFA over time without dietary change, immediately followed with continued supplementation of LC $\omega$-3PUFA, consumed concomitantly with a very low energy diet (VLED), may assist weight loss, reduced fat mass and improvement to blood biomarkers associated with possible beneficial consequences for the determinants of cardiovascular health. 
It has also been suggested that docosahexaenoic acid (DHA) has more potent anti-inflammatory effects than eicosapentaenoic acid (EPA), ${ }^{24,25}$ and that DHA is a key controller of hepatic lipid metabolism and is ultimately involved in the suppression of lipogenesis ${ }^{\mathbf{2 6}}$ which could impact on weight management. There are gender difference in the metabolism of LC $\omega$-3PUFA with studies showing significantly higher concentrations of DHA in females compared to males. ${ }^{27}$ Thus we also sought to explore possible gender differences in weight change in response to supplementation with LC $\omega$-3PUFA over time.

\section{Methods}

This was a randomised, double blind placebo controlled intervention with two parallel groups.

\section{Participants}

Obese men and women (BMI 30-40 $\mathrm{kg} \mathrm{m}^{-2}$ ), aged 18-60 years were recruited from the university campus and the general public in Newcastle, Australia to participate in a weight loss study. Excluded from the study were men and women with diagnosed type 2 diabetes, a chronic inflammatory condition, already following an energy restricted diet, allergic to fish, taking fish oil capsules or consuming two or more oily fish meals per week, also women who were pregnant or lactating.

A power calculation estimated that 16 participants per group were needed to detect a difference of approximately $2 \%$ in weight loss between the two weight loss groups at the 0.05 level of significance, with a statistical power of $80 \%$ and assuming a standard deviation of $2 \%$. Allowing for a $20 \%$ drop-out rate, 20 participants per group would be needed. When 42 participants had been recruited ( 21 in each group), recruitment was discontinued.

The study was conducted according to the guidelines laid down in the Declaration of Helsinki: ethical approval was obtained from the Human Research Ethics Committee of the University of Newcastle, Australia and written, informed consent was obtained from participants prior to commencement. The trial was registered with the Australia New Zealand Clinical Trials Registry (ACTRN12610000654055).

\section{Study design}

Participants were allocated to one of two parallel groups using computer generated numbers. Supplement containers were labelled with a different code for each group by an independent researcher before the trial commenced so that neither the principal researcher nor the participants could identify the contents of the supplements, and supplements were flavoured with peppermint to disguise identifiable tastes. For 8 weeks one group consumed $6 \times 1 \mathrm{~g}$ capsules of placebo per day (placebo group), containing sunola oil (monounsaturated oil), and the other group consumed $6 \times 1 \mathrm{~g}$ capsules of fish oil per day (fish oil group). Each fish oil capsule contained tuna oil (HiDHA, NuMega Ingredients Pty Ltd) comprising $360 \mathrm{mg}$ LC $\omega$-3PUFA with $70 \mathrm{mg}$ EPA (C20:5n-3) and $270 \mathrm{mg}$ DHA (C22:6n-3) (ratio $1: 3.9)$. Two capsules were consumed with each meal, breakfast, lunch and dinner, providing $1.62 \mathrm{~g}$ DHA and $0.42 \mathrm{~g}$ EPA per day. Both the tuna oil and the sunola oil contained the antioxidant Coviox®T-70, 3000 ppm in the tuna oil and 600 ppm in the sunola oil.

The intervention was divided into two phases. For the first phase of 4 weeks, during prior supplementation (the preparation phase), participants in the fish oil group consumed $6 \times 1 \mathrm{~g}$ capsules per day fish oil and, in the placebo group $6 \times 1 \mathrm{~g}$ capsules per day placebo, with both groups also following a healthy diet, that is their usual diet without the inclusion of fast foods. A time period of 4 weeks of supplementation to increase levels of EPA and DHA in the body was deemed appropriate because a previous study by Munro and Garg (2012) reported an almost 2-fold increase in EPA and 2.5-fold increase in DHA after 4 weeks of supplementation with LC $\omega$-3PUFA. ${ }^{28}$ A study reported similar changes after 7 weeks of supplementation with krill oil or fish oil, ${ }^{29}$ indicating that there was little apparent benefit in extending the length of initial supplementation. For the second time period of 4 weeks, the weight loss phase, participants continued consuming the supplements allocated at the commencement of the study while following a very low energy diet (VLED) of $3000 \mathrm{~kJ}$ per day using meal replacements (MRs). This phase was limited to 4 weeks because of the very restricted energy intake. For the first two weeks (weeks 5 and 6) participants received Optifast ${ }^{\circledR}$ bars and shakes to replace meals according to the Intensive Phase of the Optifast Very Low Calorie Diet Program (Novartis, Australia), supplemented daily with raw and cooked vegetables and 2 litres of drinking water. This diet provides $\geq 3000 \mathrm{~kJ}$ per day composed of $40 \%$ protein, $16 \%$ fat and $40 \%$ carbohydrate plus vitamins and minerals. At the same time, participants were given individual nutrition education and counselling, with the sessions focusing on the energy density of foods, understanding and using food labels, appropriate portion sizes and the number of portions to be consumed daily from the different food groups. This information was used to build a healthy diet using the guidelines from the Australian Guide to Healthy Eating (AGHE), ${ }^{30}$ and applied during the final two weeks of the weight loss phase (weeks 7 and 8) when the MRs were gradually phased out and healthy kilojoule controlled meals were phased in. All participants attended weekly so that weight loss progress could be monitored and to receive the next supply of meal replacements and capsules. An accredited practicing dietitian was part of the research team to advise on health care.

\section{Dietary assessment}

Prior to commencing the weight loss program, dietary intake was assessed with a 3-day food diary to determine every-day nutrient and energy intake. Participants used the weights given on food products and handy measures of cups and spoons to record food intake which was analysed for nutrient intakes using the dietary software program, Foodworks ${ }^{\circledR}$ Professional 2009, version 6 (Xyris Software (Australia) Pty Ltd) and the mean values calculated.

\section{Anthropometric assessment}

On the first day of the preparation phase, anthropometric measurements were taken in the morning after a 10-hour 
overnight fast and no alcohol consumption, with participants dressed in light clothing and without shoes. Standing height was measured to the nearest $0.1 \mathrm{~cm}$ using a stadiometer. Body weight was measured to the nearest $0.1 \mathrm{~kg}$ using a calibrated balance beam scale (PCS Measurement, NSW, Australia). BMI was calculated in kilograms $(\mathrm{kg})$ per metre squared from weight and height. Waist circumference (WC) was measured at the mid-point between the lowest rib and the top of the hipbone; the hip measurement was taken at the fullest point of the hip, as viewed from the side. The waist : hip ratio was calculated as waist girth in centimetres $(\mathrm{cm})$ divided by the hip girth (cm). Fat mass (FM), fat-free mass (FFM) and muscle mass (MM) were measured using direct segmental multifrequency bioelectrical impedance (InBody 230, Biospace Co., Ltd. Seoul, Korea). Under identical conditions, these anthropometric measurements were repeated at the beginning and the end of the weight loss phase.

\section{Biochemical analyses}

Blood sample collection. Fasting ( $\geq 10 \mathrm{~h}$ ) blood samples were collected into vacutainers, EDTA, lithium heparin and sodium fluoride, by a phlebotomist at commencement of the preparation phase and at baseline and the end of the weight loss phase. The samples were prepared by centrifuging (Heraeus Biofuge Stratos) for $10 \mathrm{~min}$ at $3000 \mathrm{~g}$ at $4{ }^{\circ} \mathrm{C}$. Plasma samples from the EDTA tubes were collected and stored at $-80^{\circ} \mathrm{C}$ awaiting further analysis. The lithium heparin and sodium fluoride vacutainers were taken to Hunter Area Pathology Services (Newcastle) for plasma analysis for C-reactive protein (CRP) and blood lipids, and for blood glucose respectively.

Plasma fatty acid analysis. The plasma samples were analysed for fatty acid composition using an acetyl chloride methylation procedure, a modification of the method of Lepage and Roy. ${ }^{31}$ Fatty acid methyl esters were quantified using a GC (Hewlett Packard 6890, Hewlett Packard, Palo Alto, CA, USA) and identified by comparing peak retention times with the retention times of synthetic standards of known fatty acid composition (Nu Check Prep, Elysian, MN, USA).

Analysis of inflammatory mediators. Enzyme-linked immunosorbent assay (ELISA) kits (R\&D Systems, Minneapolis, MN, USA) were used to analyse inflammatory markers. The assays used to determine plasma leptin and adiponectin levels employ the quantitative sandwich enzyme immunoassay technique with a detection limit of $15.6 \mathrm{pg} \mathrm{ml}^{-1}$ and $3.9 \mathrm{ng} \mathrm{m} \mathrm{m}^{-1}$ respectively and inter- and intra-assay coefficients of variance (CVs) of less than $5.5 \%$ and $7 \%$ respectively. High sensitivity ELISA kits were used to determine levels of tumour necrosis factor- $\alpha$ (hs-TNF- $\alpha$ ) and interleukin6 (hs-IL-6). Minimal detectable concentration of these kits was $0.106 \mathrm{pg} \mathrm{ml}^{-1}$ and $0.039 \mathrm{pg} \mathrm{ml}^{-1}$ respectively with an intra- and inter-assay CVs of $<9 \%$. Analysis of high-sensitivity C-reactive protein (hs-CRP) was conducted using an immunoturbidimetric method (Hunter Area Pathology Service, Newcastle, NSW, Australia) with a minimal detection level of $0.15 \mathrm{mg} \mathrm{l}^{-1}$.

\section{Statistical analyses}

For anthropometric measurements, mixed design 3-way ANOVA was used to measure 3-way interactions between group, time and gender. Within group changes from baseline were determined using paired samples $t$-test and ANOVA was used to test group mean differences. Pearson product-normal correlations $(r)$ were used to show relationships. Data are presented as mean values and standard error of mean (SEM), with $P \leq 0.05$ indicating significance. All statistical analyses were carried out with SPSS software (version 19.0, SPSS Inc., Chicago, IL, USA).

\section{Results}

Of the 42 participants who commenced the study, 39 completed the 8-week intervention, 20 in the fish oil group (FO) (15 females, 5 males) and 19 in the placebo group (PB) (15 females, 4 males). Of the 3 who did not complete; 2 withdrew in week 2 for family reasons and one did not keep appointments for measurements and blood collection. Five extreme outliers were identified for hs-CRP ( $n=2$ PB and 3 FO) and were excluded from the analysis of this biomarker.

\section{Diet}

The mean daily energy intake of participants was similar for the two groups with $8293 \pm 2597 \mathrm{~kJ}$ per day for FO and $8727 \pm 2469 \mathrm{~kJ}$ per day for PB. At commencement of the study the percentage fatty acid composition of plasma lipids showed no significant difference between the two groups. After supplementation during the preparation phase with LC $\omega$-3PUFA in the FO group, EPA and DHA had increased almost 2-fold from week 0 to week 4 $(P<0.001$ for both), and they were significantly higher than the levels in the PB group $(P<0.001$ for both $)$ (Fig. 1). There were very modest changes to levels of EPA and DHA after the weight loss dietary regimen (week 8) and they remained significantly higher in the FO group compared to the PB group $(P<0.001$ for both).

\section{Anthropometric measurements}

At commencement of the study the baseline measurements for weight, BMI, FM, FFM, MM, WC and hip were not significantly

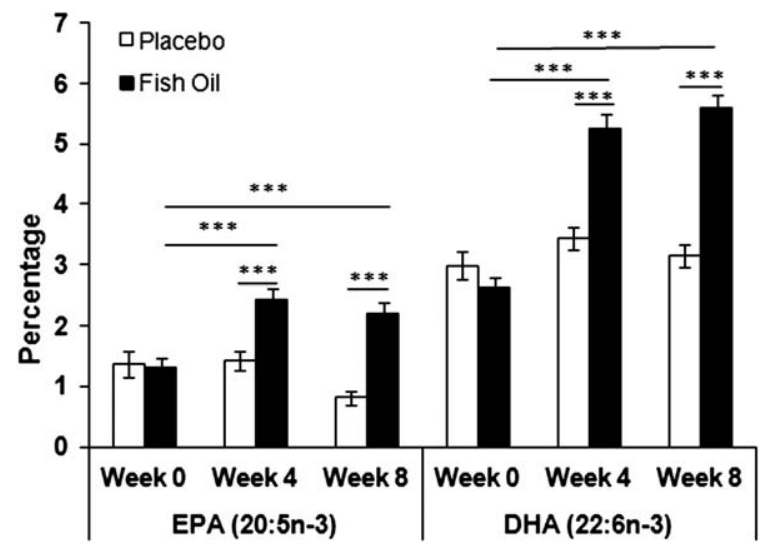

Fig. 1 Baseline values and percent changes for LC $\omega$-3PUFA at pre-supplementation and weight loss phases ( 0,4 and 8 weeks). (Mean values \pm SEM), $* * * P<0.001$. 
different between the two groups, PB and FO, and at the end of the 4 weeks preparation phase anthropometric measurements were relatively unchanged (Table 1). After the 4 -week weight loss phase with continued supplementation with LC $\omega$-3PUFA in the FO group there was a significant reduction in body weight $-7.01 \%$ ( $-6.12 \mathrm{~kg}), \mathrm{FM}-13.6 \%(-4.36 \mathrm{~kg}), \mathrm{FFM}-3.2 \%(-1.68$ $\mathrm{kg})$, WC $-5.8 \%(-5.69 \mathrm{~cm})$, and hip measurement $-3.3 \%$ $(-3.78 \mathrm{~cm})(P<0.001$ for all). Also, after continued supplementation with placebo in the $\mathrm{PB}$ group there were significant reductions in, body weight $-6.2 \%(-5.79 \mathrm{~kg}), \mathrm{FM}-11.9 \%$ $(-4.46 \mathrm{~kg})$, WC $-5.3 \%(-5.68 \mathrm{~cm})$, and hip measurement $-3.3 \%(-3.82 \mathrm{~cm})(P<0.001$ for all $)$, and in FFM $-2.3 \%(-1.33$ kg) $(P<0.01)$ (Table 1).

As gender differences in the metabolism of LC $\omega$-3PUFA have been reported ${ }^{27}$ mixed design 3-way ANOVA was used to investigate the interaction between the FO and PB groups by gender over time for changes to anthropometric measurements. A significant 3-way interaction between time, group and gender was observed for percentage weight loss, $F(1,35)=5.55$, $P=0.024$, and for percentage decrease to BMI, $F(1,35)=5.3$, $P=0.027$, but no significance in decreased $\mathrm{FM}, F(1,35)=2.27$, $P=0.14$; WC, $F(1,35)=0.31, P=0.58$; hip measurement, $F(1,35)$ $=2.56, P=0.119$. Analysis of the interaction between group and gender over time also revealed that in the FO group there was a greater, but non-significant percentage weight loss in females compared to males $(-7.21 \% v s$. $-6.3 \%$ respectively), and in the PB group there was a greater, but non-significant percentage weight loss in males compared to females $(-7.69 \%$ vs. $-5.82 \%$ respectively). There were, however, significant differences in weight and BMI change between females in FO and PB, with a significantly greater percentage decrease for females in $\mathrm{FO}$ compared to females in PB for weight $(-7.21 \%$ vs. $-5.82 \%)$ and BMI $(-7.43 \%$ vs. $-5.91 \%)$ respectively $(P<0.05$ for both) (Table 2 details anthropometric results for females).

Correlations between changes in anthropometric measurements and LC $\omega$-3PUFA were computed. There was a negative and non-significant correlation between percentage weight change and EPA, $r=-0.316(P=0.175)$, and DHA, $r=-0.283$ $(P=0.227)$.

\section{Blood biomarkers}

The changes in blood biomarkers from baseline to the end of the 4 week preparation phase were very modest and nonsignificant for both groups apart from TGs which showed a significant reduction in the FO group $(P<0.05)$ (Table 3$)$.

At week 8, after 4 weeks of a VLED and continued supplementation with LC n-3 PUFA, there were significant changes to blood biomarkers within each group. For both FO and PB there was a significant reduction in total cholesterol (TC) $(P<0.001$ for both). In FO there were significant reductions in high density lipoprotein-cholesterol (HDL-C) and low density lipoprotein-cholesterol (LDL-C) $(P<0.001$ for both) and in triglycerides (TG)s and glucose $(P<0.01$ for both). For PB there were significant reduction in HDL-C, LDL-C and TGs $(P<0.01$ for all $)$ but not glucose (Table 3). The only significant reduction observed in the inflammatory mediators was for leptin $(P<$

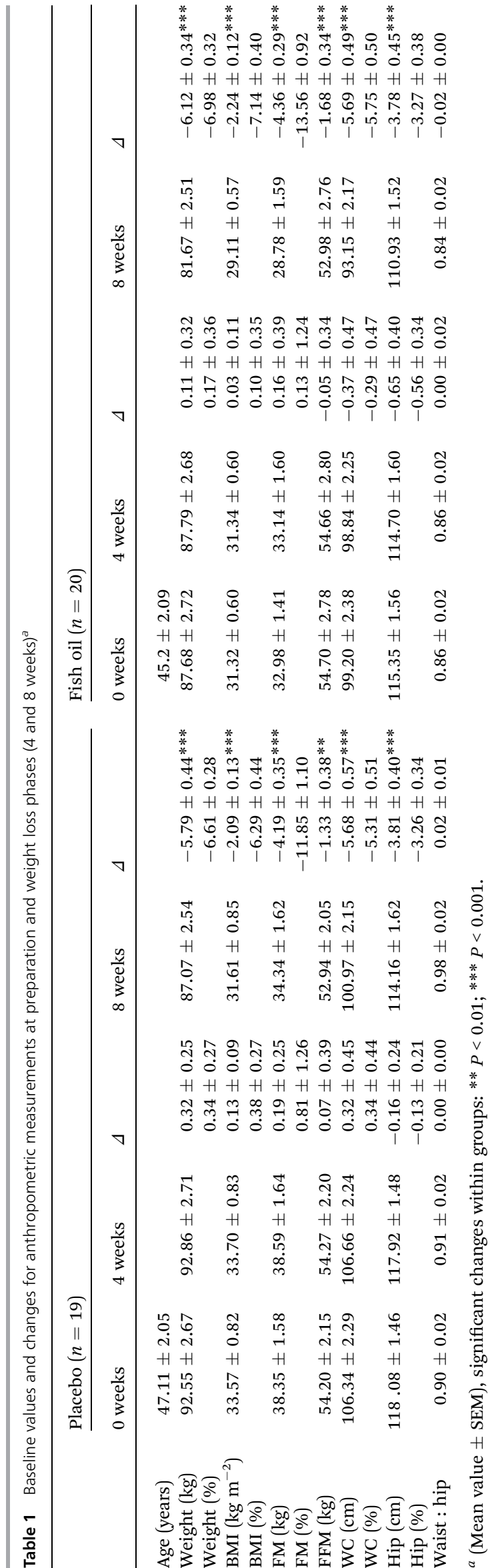




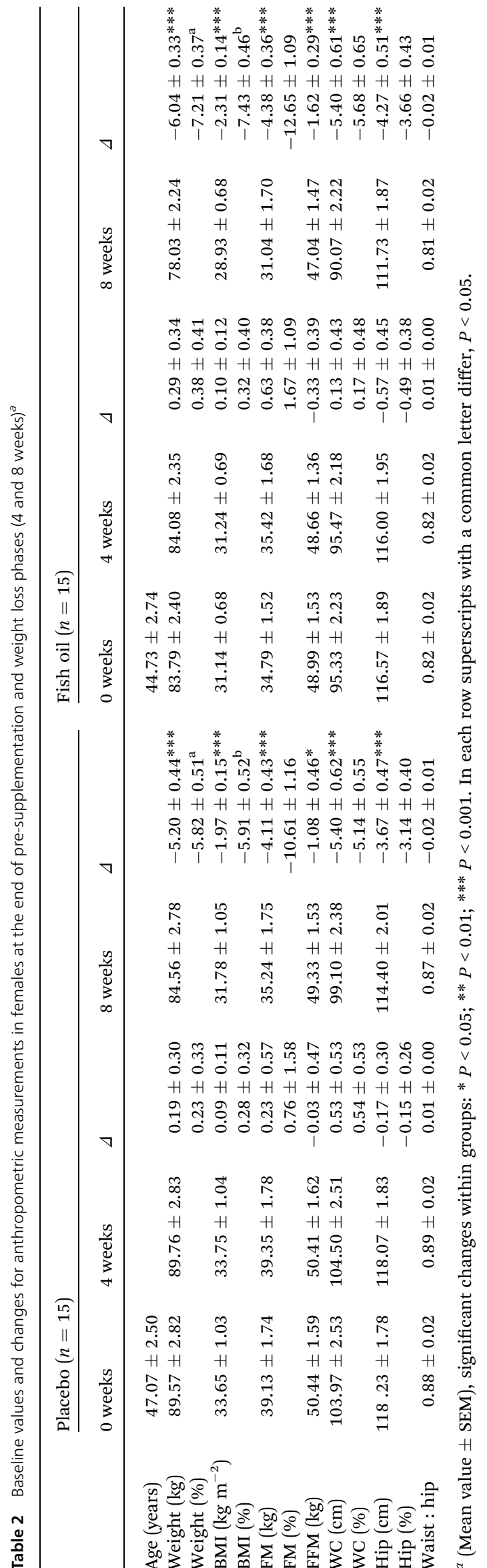

0.001 for both FO and PB). Differences between the two groups were not significant.

\section{Discussion}

Despite compliance with supplement consumption, as evidenced by the significant increase in levels of EPA and DHA in the FO group, anthropometric measurements were relatively unchanged in both FO and PB at the end of the 4 week preparation phase, with no significant differences between the two groups, suggesting that, within this short time-frame, LC $\omega$ 3PUFA consumed without dietary energy restriction had no effect on body weight or FM. In the subsequent 4-week weight loss phase with continued supplementation, dietary compliance was facilitated with the use of MRs which have a satiating effect that lasts for between 3 and 5 hours after consumption. ${ }^{32}$ The significant reductions in all anthropometric measurements within each group at the end of the weight loss phase suggest the effectiveness of the VLED. The greater, but non-significant percentage weight loss in females compared to males $(-7.21 \%$ vs. $-6.3 \%$ respectively) in the FO group suggests a possible effect on the females from the higher concentrations of DHA in the LC $\omega$-3PUFA supplements. The greater, but non-significant percentage weight loss in males compared to females $(-7.69 \%$ vs. $-5.82 \%$ respectively) in the PB group could be attributed to the suggestion that females lose less weight than males with a comparable degree of energy restriction. ${ }^{33}$ The possible beneficial effects of DHA on weight loss observed in females compared to males should be treated with caution because of the low number of males involved in this study and more research on these potential differences is suggested. The significantly greater percentage decrease in weight $(-7.21 \%$ vs. $-5.82 \%)$ and BMI $(-7.43 \%$ vs. $-5.91 \%)$ in females in FO compared to females in PB $(P<0.05$ for both) could have been influenced by the higher levels of DHA (1.62 $\mathrm{g}$ per day) compared to EPA ( 0.42 $\mathrm{g}$ per day) with DHA/EPA ratio of $1: 3.9$ in the supplement used in this study. A study by Kunesova et al. (2006) on LC $\omega$-3PUFA enhanced weight loss in extremely obese women following a VLED reported a significant correlation between a decrease in BMI and DHA, but not EPA, suggesting that DHA appeared to be the active component in enhanced weight loss in obese females. ${ }^{15}$

It is becoming apparent that there are gender differences in the metabolism of LC $\omega$-3PUFA. It has previously been reported that not only are there higher levels of DHA and lower levels of EPA in serum lipids in females compared to males, ${ }^{27}$ but females are more responsive to the metabolism of LC $\omega$-3PUFA compared to males. ${ }^{27,34}$ Other studies have also reported that females had a higher percentage of total fatty acids as DHA in plasma $^{35}$ and adipose lipids ${ }^{36}$ compared to males.

The mechanisms by which LC $\omega$-3PUFA assist the reduction of body fat and/or body weight are still being explored. It has been suggested that LC $\omega$-3PUFA modulates lipid metabolism promoting lipolysis and enhancing hepatic fatty acid oxidation, inhibiting fatty acid synthesis and VLDL secretion. ${ }^{26,37}$ According to Jump et al. (2008) it has been established that DHA is a key controller of hepatic lipid synthesis, has a major impact on 
$\nabla$

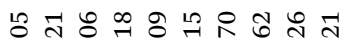

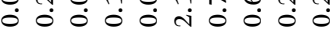

䒘유 \%

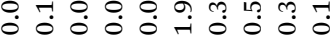
$\mathrm{H}+\mathrm{H} H \mathrm{H} H \mathrm{H} H \mathrm{H} H$

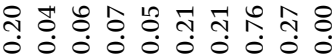

4

궁 옹

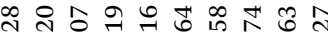
000000 $\mathrm{H}+\mathrm{H}+\mathrm{H} H+\mathrm{H} H+\mathrm{H}+$

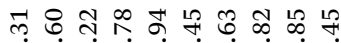

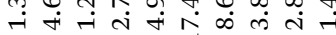

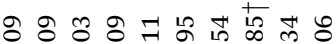

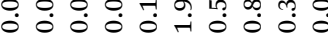
$\mathrm{H} H \mathrm{H} H \mathrm{H} H \mathrm{H} H \mathrm{H} H$ ને

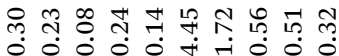

$H+H+H+H+H+H$

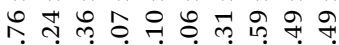
ำ

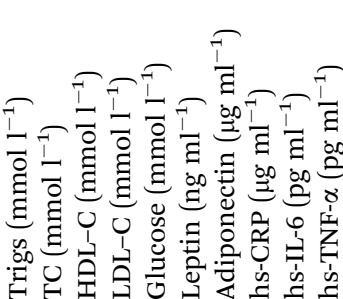

hepatic lipid metabolism and is ultimately involved in the suppression of lipogenesis. ${ }^{26}$ This suggests a possible explanation for the greater reduction in weight experienced by the women in the FO group in this study.

A limited number of previous studies have reported on the effects of combined supplementation with LC $\omega$-3PUFA and dietary energy restriction for weight loss over a time of 8 weeks or longer. Energy restricted or controlled diets combined with supplementation with LC $\omega$-3PUFA in different ratios and time frames such as DHA : EPA of $2.2: 1$ (2.9 $\mathrm{g}$ per day DHA and $1.3 \mathrm{~g}$ per day EPA) over 12 weeks, ${ }^{5}$ DHA : EPA of $1: 1.5$ (0.72 g per day DHA and $1.08 \mathrm{~g}$ per day EPA) over 8 weeks, ${ }^{18}$ and DHA : EPA of $1: 5$ ( $3 \mathrm{~g}$ per day EPA + DHA) over 24 weeks in combination with exercise, ${ }^{19}$ all reported no significant difference in weight reduction compared to the control, although Kabir et al. (2007) did report a significantly greater decrease in FM in the treatment group compared to the control. ${ }^{18}$ These studies varied greatly with respect to dosage of LC $\omega$-3PUFA, the EPA : DHA ratio, duration of supplementation, the health status of the study participants and gender of the groups and it is, therefore, difficult to draw any firm conclusions from these studies.

It is possible that an extended period of LC $\omega$-3PUFA intake during the growth years rather than dietary supplementation with large doses over a short duration in adults influences body weight. In this context, several studies have reported a significantly higher concentration of LC $\omega$-3PUFA in normal weight compared to obese individuals ${ }^{\mathbf{2 0 - 2 3}}$ but timing and duration of intake of LC $\omega$-3PUFA in the individuals is unknown. It is possible that incorporation of LC $\omega$-3PUFA into the adipose tissue is needed for any effects on weight loss to occur and, despite long-term supplementation in adults, increase in the levels of EPA and DHA in adipose tissue is reportedly modest. ${ }^{5}$ As late foetal and early postnatal life is a highly sensitive period when adipose tissue expands rapidly ${ }^{38-40}$ this could be a critical window of opportunity to improve the balance of fatty acids involved in adipogenesis and lipogenesis. A study in humans, where mothers received DHA supplementation from 21 weeks gestation until the end of the third month of lactation, reported a significant time-dependent effect of DHA on weight and BMI reduction in their infants at 21 months. ${ }^{41}$ Thus, it would appear that when increasing levels of LC $\omega$-3PUFA for weight management, gender, age, duration of supplementation, concentration and ratio of EPA : DHA all require further consideration.

Weight loss is associated with reductions in blood lipids which is beneficial to health. ${ }^{\mathbf{4 2}}$ Accordingly, after weight loss this study showed significant reductions in levels of TGs, TC and LDL-C within both FO and PB, with no significant difference between the two groups. There was, however, also a significant reduction in TG in the FO group, but not in the PB group, after the preparation phase Supplementation with LC $\omega$ 3PUFA is firmly associated with a reduction in TG levels ${ }^{43-46}$ which supports our finding. The subsequent significant reduction in TG in both groups after the weight loss phase could be as a result of the considerable weight reduction experienced by both groups over the 4 weeks, though continued supplementation LC $\omega$-3PUFA did influence a greater reduction in TG in the FO group. Reductions in TC and 
LDL-C were also greater in FO than PB but, again, the differences were modest and not significant. It has been suggested that LC $\omega$-3PUFA has no effect on TC levels ${ }^{\mathbf{4 4 , 4 7}}$ and opinions on the effect of LC $\omega$-3PUFA on LDL-C are inconclusive. Studies have reported that supplementation with LC $\omega$-3PUFA resulted in small increases in LDL-C levels. ${ }^{\mathbf{4 8 , 4 9}}$ However, it has been suggested that an increase in LDL-C is accompanied by an increase in the size of the LDL particles which are thought to be less atherogenic. ${ }^{\mathbf{5 0}}$

A reduction in HDL-C also occurred in both FO and PB which is not desirable because it is known to have cardioprotective effects. ${ }^{51}$ Reports on the effect of weight loss on levels of HDL-C are conflicting; some studies have shown small increases, ${ }^{52-54}$ some have reported small decreases, ${ }^{55,56}$ and others have shown no changes at all. ${ }^{57,58}$ It would appear that HDL-C levels reduce during the period when weight is decreasing, as occurred in this study. It has been suggested that during a decrease in weight, a reduction in triglyceride rich lipoprotein synthesis impairs VLDL-C catabolism with a subsequent reduction in the transfer of lipids to HDL-C. When weight stabilises, lipoprotein lipase increases and with it the hydrolysis of VLDL-C and a resumption in the transfer of lipids to HDL-C. ${ }^{42}$ From a review of studies, Dattilo and Kris-Etherton (1992) reported a mean decrease in HDL-C during actual weight loss which then increased when a stable weight was achieved. ${ }^{42}$ A study by Munro and Garg (2012) with an identical weight loss diet conducted over the same length of time as the current study, also reported a decrease in HDL-C levels at the end of the weight loss phase. Then, during the 10 weeks of maintenance that followed, HDL-C levels increased, reaching levels higher than those recorded at baseline. ${ }^{28}$ Studies have also shown that LC $\omega$-3PUFA, particularly DHA, increases HDL-C concentrations ${ }^{59}$ but, despite the greater concentration of DHA in the supplementation with LC $\omega$-3PUFA in this study, HDL-C levels did not increase. It is possible that this occurred as a consequence of the considerable weight loss, as discussed above.

Apart from leptin, the changes to inflammatory mediators in this study were extremely modest. After 4 weeks of prior supplementation with LC $\omega$-3PUFA and with virtually no weight change, there was an extremely modest increase in leptin for FO and reduction for PB. However, after the 4 week weight loss phase there was a significant reduction in leptin for both FO and $\mathrm{PB}$ ( $64 \%$ and $57 \%$ respectively) but no significant difference between the two groups, suggesting that weight loss and not LC $\omega$-3PUFA influenced the changes. The magnitude of these reductions is similar to the findings in other studies with similar energy restricted diets but no supplementation with LC $\omega$-3PUFA. ${ }^{60-62}$ There is conflicting evidence on the effect of LC $\omega$-3PUFA on plasma leptin levels, ${ }^{63}$ with one study reporting that EPA increases leptin levels,${ }^{64}$ another suggesting that LC $\omega$ 3PUFA reduces leptin levels. ${ }^{65}$ The changes to adiponectin levels were very modest with no distinct trend for weight loss and supplementation with LC $\omega$-3PUFA. Madsen et al. (2008) reported an increase in levels of adiponectin in response to diet induced weight loss but suggest that a reduction in weight of at least $10 \%$ is required to show a significant increase in levels of adiponectin. ${ }^{66}$
After the 4 weeks of prior supplementation CRP levels increased for FO and decreased for PB. However, after 4 weeks of weight loss there was an almost identical decrease in CRP for both groups. Conflicting findings have been reported from studies investigating the effect of diet and/or weight on CRP levels. One study reported that LC $\omega$-3PUFA and not weight loss was associated with a significant reduction in $\mathrm{CRP},{ }^{67}$ while another study reported an inverse association with LC $\omega$-3PUFA and serum CRP in men. ${ }^{68}$ Observational studies have reported that LC $\omega$-3PUFA is associated with lower levels of CRP, ${ }^{69,70}$ while randomised control trials have reported no effect of LC $\omega$-3PUFA on CRP levels in healthy people. ${ }^{71,72}$ It has also been suggested that a reduction in the level of CRP is directly related to a reduction in weight. ${ }^{73}$ However, it would appear that a significant reduction in the long-term requires weight loss to be greater than $10 \%{ }^{66}$ and it is possible that not only differences in the length of the intervention, but differences in ages of subjects within the groups and components of the different diets could influence levels of plasma CRP. ${ }^{63}$

After the preparation phase and after the weight loss phase there were no significant differences in changes to IL-6 and TNF- $\alpha$ either within groups or between groups, suggesting that LC $\omega$-3PUFA had no effect. It has been reported that dietary supplementation with LC $\omega$-3PUFA can suppress the production of TNF- $\alpha,^{74}$ however no significant changes in IL-6 and TNF $\alpha$ were observed in a study when subjects received either $2 \mathrm{~g}, 4 \mathrm{~g}$ or $6 \mathrm{~g}$ per day of Hi DHA (26\% DHA and 6\% EPA) over 12 weeks. $^{75}$ Similar findings were reported in a study that did not observe any effect on levels of IL-6 concentrations after supplementation with $3.4 \mathrm{~g}$ per day of LC $\omega$-3PUFA for 6 weeks. ${ }^{76}$ Another study reported that weight loss and not LC $\omega$-3PUFA influenced a significant reduction in IL-6 and a non-significant reduction in TNF- $\alpha{ }^{77}$ These authors suggest that as IL- 6 is secreted by adipose tissue, a reduction in FM could contribute to a reduction of IL-6 levels.

\section{Conclusion}

It has been suggested that compared to males, females are more responsive to the metabolism of LC $\omega$-3PUFA, possibly because of the hormone oestrogen and they therefore benefit from enhanced thermogenesis from LC $\omega$-3PUFA. Although a greater percentage reduction in weight and BMI was observed in females in the FO group, because of the small number of participants, particularly of males, the results should be treated with caution. The findings suggest that further research over a longer period of time is needed to investigate gender differences in the potential link between LC $\omega$-3PUFA, including DHA and EPA on adiposity and weight.

\section{Limitations}

Although sufficient participants completed the study to detect significant differences in anthropometric measurements, the sample size was small. Overall, changes to blood biomarkers were greater in the fish oil group and, with a larger sample size, these differences could have been significant. 


\section{Conflict of interest}

The authors have no conflict of interest to disclose.

\section{Acknowledgements}

NuMega Ingredients Pty Ltd, Australia, supplied the fish oil and sunola oil capsules. Nestle Nutrition, Australia, supplied the Optifast ${ }^{\circledR}$ bars and shakes for the meal replacements.

\section{References}

1 WHO, Overweight and obesity, Fact sheet no. 311, accessed 29 February 2012.

2 NCEP, Circulation, 2002, 106, 3143-3421.

3 P. Trayhurn, Endocrinology, 2005, 146, 1003-1005.

4 A. H. Berg and P. E. Scherer, Circ. Res., 2005, 96, 939-949.

5 J. D. Krebs, L. M. Browning, N. K. McLean, J. L. Rothwell, G. D. Mishra, C. S. Moore and S. A. Jebb, Int. J. Obes., 2006, 30, 1535-1544.

6 P. M. Kris-Etherton, W. S. Harris and L. J. Appel, Arterioscler., Thromb., Vasc. Biol., 2003, 23, e20-30.

7 P. C. Calder, Clin. Sci., 2004, 107, 1-11.

8 P. Flachs, O. Horakova, P. Brauner, M. Rossmeisl, P. Pecina, N. Franssen-van Hal, J. Ruzickova, J. Sponarova, Z. Drahota, C. Vlcek, J. Keijer, J. Houstek and J. Kopecky, Diabetologia, 2005, 48, 2365-2375.

9 J. Ruzickova, M. Rossmeisl, T. Prazak, P. Flachs, J. Sponarova, M. Veck, E. Tvrzicka, M. Bryhn and J. Kopecky, Lipids, 2004, 39, 1177-1185.

10 R. C. LeBoeuf and M. S. Veldee, J. Nutr., 1993, 123, 547-558. 11 D. A. Pan and L. H. Storlien, J. Nutr., 1993, 123, 512-519.

12 A. D. Kriketos, R. M. Robertson, T. A. Sharp, H. Drougas, G. W. Reed, L. H. Storlien and J. O. Hill, J. Hypertens., 2001, 19, 1745-1754.

13 A. Minami, N. Ishimura, S. Sakamoto, E. Takishita, K. Mawatari, K. Okada and Y. Nakaya, Br. J. Nutr., 2002, 87, 157-162.

14 T. Raclot, R. Groscolas, D. Langin and P. Ferre, J. Lipid Res., 1997, 38, 1963-1972.

15 M. Kunesova, R. Braunerova, P. Hlavaty, E. Tvrzicka, B. Stankova, J. Skrha, J. Hilgertova, M. Hill, J. Kopecky, M. Wagenknecht, V. Hainer, M. Matoulek, J. Parizkova, A. Zak and S. Svacina, Physiol. Res., 2006, 55, 63-72.

16 I. Thorsdottir, H. Tomasson, I. Gunnarsdottir, E. Gisladottir, M. Kiely, M. D. Parra, N. M. Bandarra, G. Schaafsma and J. A. Martinez, Int. J. Obes., 2007, 31, 1560-1566.

17 C. Couet, J. Delarue, P. Ritz, J. M. Antoine and F. Lamisse, Int. J. Obes. Relat. Metab. Disord., 1997, 21, 637-643.

18 M. Kabir, G. Skurnik, N. Naour, V. Pechtner, E. Meugnier, S. Rome, A. Quignard-Boulange, H. Vidal, G. Slama, K. Clement, M. Guerre-Millo and S. W. Rizkalla, Am. J. Clin. Nutr., 2007, 86, 1670-1679.

19 L. F. DeFina, L. G. Marcoux, S. M. Devers, J. P. Cleaver and B. L. Willis, Am. J. Clin. Nutr., 2011, 93, 455-462.

20 M. Micallef, I. Munro, M. Phang and M. Garg, Br. J. Nutr., 2009, 102, 1370-1374.
21 M. Karlsson, S. Marild, J. Brandberg, L. Lonn, P. Friberg and B. Strandvik, Obesity, 2006, 14, 1931-1939.

22 C. Klein-Platat, J. Drai, M. Oujaa, J. L. Schlienger and C. Simon, Am. J. Clin. Nutr., 2005, 82, 1178-1184.

23 S. Scaglioni, E. Verduci, M. Salvioni, M. G. Bruzzese, G. Radaelli, R. Zetterstrom, E. Riva and C. Agostoni, Acta Paediatr., 2006, 95, 964-969.

24 G. V. Halade, M. M. Rahman, A. Bhattacharya, J. L. Barnes, B. Chandrasekar and G. Fernandes, J. Immunol., 2010, 184, 5280-5286.

25 E. Oliver, F. C. McGillicuddy, K. A. Harford, C. M. Reynolds, C. M. Phillips, J. F. Ferguson and H. M. Roche, J. Nutr. Biochem., 2012, 23, 1192-1200.

26 D. B. Jump, D. Botolin, Y. Wang, J. Xu, O. Demeure and B. Christian, Chem. Phys. Lipids, 2008, 153, 3-13.

27 F. L. Crowe, C. M. Skeaff, T. J. Green and A. R. Gray, Br. J. Nutr., 2008, 99, 168-174.

28 I. A. Munro and M. L. Garg, Br. J. Nutr., 2012, 108, 14661474.

29 S. M. Ulven, B. Kirkhus, A. Lamglait, S. Basu, E. Elind, T. Haider, K. Berge, H. Vik and J. I. Pedersen, Lipids, 2011, 46, 37-46.

30 E. Kellet, A. E. Smith and Y. Schmerlaib, The Australian Guide to Healthy Eating, Australian Government Department of Health and Ageing, Canberra, 1998.

31 G. Lepage and C. C. Roy, J. Lipid Res., 1986, 27, 114-120.

32 D. Q. Rothacker and S. Watemberg, Int. J. Food Sci. Nutr., 2004, 55, 223-226.

33 A. Sartorio, N. A. Maffiuletti, F. Agosti and C. L. Lafortuna, J. Endocrinol. Invest., 2005, 28, 494-501.

34 G. C. Burdge and S. A. Wootton, Br. J. Nutr., 2002, 88, 411420.

35 E. Dewailly, C. Blanchet, S. Lemieux, L. Sauve, S. Gingras, P. Ayotte and B. J. Holub, Am. J. Clin. Nutr., 2001, 74, 464473.

36 R. Tavendale, A. J. Lee, W. C. Smith and H. Tunstall-Pedoe, Atherosclerosis, 1992, 94, 161-169.

37 C. C. Tai and S. T. Ding, J. Nutr. Biochem., 2010, 21, 357-363. 38 G. Ailhaud and P. Guesnet, Obes. Rev., 2004, 5, 21-26.

39 J. L. Knittle, K. Timmers, F. Ginsberg-Fellner, R. E. Brown and D. P. Katz, J. Clin. Invest., 1979, 63, 239-246.

40 B. Muhlhausler and S. R. Smith, Trends Endocrinol. Metab., 2009, 20, 51-57.

41 R. Lucia Bergmann, K. E. Bergmann, E. Haschke-Becher, R. Richter, J. W. Dudenhausen, D. Barclay and F. Haschke, J. Perinat. Med., 2007, 35, 295-300.

42 A. M. Dattilo and P. M. Kris-Etherton, Am. J. Clin. Nutr., 1992, 56, 320-328.

43 L. Hooper, R. L. Thompson, R. A. Harrison, C. D. Summerbell, H. Moore, H. V. Worthington, P. N. Durrington, A. R. Ness, N. E. Capps, G. Davey Smith, R. A. Riemersma and S. B. Ebrahim, Cochrane Database Syst. Rev., 2007, CD003177.

44 W. S. Harris, J. Lipid Res., 1989, 30, 785-807.

45 W. S. Harris and D. Bulchandani, Curr. Opin. Lipidol., 2006, 17, 387-393.

46 T. A. Jacobson, Am. J. Clin. Nutr., 2008, 87, 1981S-1990S. 
47 E. M. Balk, A. H. Lichtenstein, M. Chung, B. Kupelnick, P. Chew and J. Lau, Atherosclerosis, 2006, 189, 19-30.

48 M. A. Micallef and M. L. Garg, J. Nutr., 2008, 138, 1086-1090. 49 M. A. Micallef and M. L. Garg, Atherosclerosis, 2009, 204, 476482.

50 W. E. Connor, J. Am. Diet. Assoc., 1968, 52, 202-208.

51 W. E. Boden, Am. J. Cardiol., 2000, 86, 19L-22L.

52 P. Marckmann, S. Toubro and A. Astrup, Eur. J. Clin. Nutr., 1998, 52, 329-333.

53 D. E. Wilson and R. S. Lees, J. Clin. Invest., 1972, 51, 10511057.

54 R. R. Wing, E. Venditti, J. M. Jakicic, B. A. Polley and W. Lang, Diabetes Care, 1998, 21, 350-359.

55 M. Flechtner-Mors, H. H. Ditschuneit, T. D. Johnson, M. A. Suchard and G. Adler, Obes. Res., 2000, 8, 399-402.

56 A. Weltman, S. Matter and B. A. Stamford, Am. J. Clin. Nutr., 1980, 33, 1002-1009.

57 A. Poobalan, L. Aucott, W. C. Smith, A. Avenell, R. Jung, J. Broom and A. M. Grant, Obes. Rev., 2004, 5, 43-50.

58 J. Hauptman, C. Lucas, M. N. Boldrin, H. Collins and K. R. Segal, Arch. Fam. Med., 2000, 9, 160-167.

59 T. A. Jacobson, S. B. Glickenstein, J. D. Rowe and P. N. Soni, J. Clin. Lipidol., 2012, 6, 5-18.

60 B. E. Wisse, L. A. Campfield, E. B. Marliss, J. A. Morais, R. Tenenbaum and R. Gougeon, Am. J. Clin. Nutr., 1999, 70, 321-330.

61 G. R. Dubuc, S. D. Phinney, J. S. Stern and P. J. Havel, Metabolism, 1998, 47, 429-434.

62 I. A. Munro and M. L. Garg, Food Funct., 2011, 2, 611-616.

63 M. J. Puglisi, A. H. Hasty and V. Saraswathi, J. Nutr. Biochem., 2011, 22, 101-108.

64 P. Perez-Matute, A. Marti, J. A. Martinez, M. P. FernandezOtero, K. L. Stanhope, P. J. Havel and M. J. Moreno-Aliaga, Am. J. Physiol.: Regul. Integr. Comp. Physiol., 2005, 288, R1682-R1688.
65 J. Ukropec, J. E. Reseland, D. Gasperikova, E. Demcakova, L. Madsen, R. K. Berge, A. C. Rustan, I. Klimes, C. A. Drevon and E. Sebokova, Lipids, 2003, 38, 10231029.

66 E. L. Madsen, A. Rissanen, J. M. Bruun, K. Skogstrand, S. Tonstad, D. M. Hougaard and B. Richelsen, Eur. J. Endocrinol., 2008, 158, 179-187.

67 P. D. Tsitouras, F. Gucciardo, A. D. Salbe, C. Heward and S. M. Harman, Horm. Metab. Res., 2008, 40, 199-205.

68 I. Reinders, J. K. Virtanen, I. A. Brouwer and T. P. Tuomainen, Eur. J. Clin. Nutr., 2012, 66, 736-741.

69 R. Farzaneh-Far, W. S. Harris, S. Garg, B. Na and M. A. Whooley, Atherosclerosis, 2009, 205, 538-543.

70 E. Lopez-Garcia, M. B. Schulze, J. E. Manson, J. B. Meigs, C. M. Albert, N. Rifai, W. C. Willett and F. B. Hu, J. Nutr., 2004, 134, 1806-1811.

71 A. Geelen, I. A. Brouwer, E. G. Schouten, C. Kluft, M. B. Katan and P. L. Zock, Eur. J. Clin. Nutr., 2004, 58, 1440-1442.

72 A. C. Skulas-Ray, P. M. Kris-Etherton, W. S. Harris, J. P. Vanden Heuvel, P. R. Wagner and S. G. West, Am. J. Clin. Nutr., 2011, 93, 243-252.

73 E. Selvin, N. P. Paynter and T. P. Erlinger, Arch. Intern. Med., 2007, 167, 31-39.

74 G. E. Caughey, E. Mantzioris, R. A. Gibson, L. G. Cleland and M. J. James, Am. J. Clin. Nutr., 1996, 63, 116-122.

75 A. M. Coates, C. M. Milte, J. D. Buckley, A. Ferrante and P. R. C. Howe, Reduced production of inflammatory cytokines accompanies fish oil-induced increases of the omega-3 index, 35th Annual Scientific Meeting of Nutrition Society of Australia and Nutrition Society of New Zealand, Queenstown, New Zealand, 2011.

76 D. C. Chan, G. F. Watts, P. H. Barrett, L. J. Beilin and T. A. Mori, Clin. Chem., 2002, 48, 877-883.

77 A. Jellema, J. Plat and R. P. Mensink, Eur. J. Clin. Invest., 2004, 34, 766-773. 